\title{
Cross-Leg Flap: Its Reliability and Outcome
}

\author{
Mohamed A. Shoeib \\ Plastic Surgery, Sohag University, Sohag, Egypt. \\ Email: mrahmoni@yahoo.com \\ Received August $2^{\text {nd }}, 2012$; revised September $5^{\text {th }}, 2012$; accepted October $7^{\text {th }}, 2012$
}

\begin{abstract}
Background: Cross-leg flaps for lower limb wound coverage have been replaced by free tissue transfer in the last two decades. However, there are certain difficult situations where the free flap cannot be employed and alternative methods are needed. We describe our experience with cross-leg flap in 135 patients for the reconstruction of difficult leg defects in which no possible other options available for reconstruction of the defect. Materials and Methods: This study was carried upon one hundred thirty five patients suffering from leg and/or foot soft tissue injury. This study focused on the cases that need coverage of bone, tendons, and neurovascular bundle, while those that managed by local flap or split thickness graft were excluded. Results: All patients ambulate well after 5 - 10 days, except for 14 patients who had associated orthopedic problems as external fixation, or other fractures. Cosmetic outcome were excellent in all patients, but 3 patient's mothers complaint of bulky flap, and for them liposuction was done with postoperative satisfaction. 2 cases complaint of early donnar sit graft, but later on they were satisfied. Conclusion: Cross-leg flap offers the possibility of salvaging limbs that are otherwise nonreconstructable.
\end{abstract}

Keywords: Cross-Leg; Reconstruction; Trauma; Separation

\section{Introduction}

Severe trauma such as Gustilo type III-C injuries of lower leg caused by accidents happens more often than before. These injuries are usually accompanied with extensive damage of the soft tissue, severe comminuted fractures, or bone defects, and even with injuries of nerves and main vessels. The reconstruction of limb salvage in these cases is a great challenge for orthopedic and plastic surgeons [1].

Composite tissue loss in extremities involving neurovascular structures has been a major challenge for reconstructive surgeons. Principles of reconstruction cascade dictate first using local and regional tissues. However, local and regional flaps may not be suitable for larger defects, and adequate fresh tissue can only be obtained with microsurgical procedures. The success of free flap operations depends on the presence of healthy recipient vessels. In cases with no suitable recipient artery and vein or in those where even the use of vein grafts would not be feasible, the lower limb could be salvaged through a cross-leg flap procedure.

The cross leg flaps have stood the test of time ever since its first description by Hamilton in 1854 [2]. It has always found an important place in the surgeon's armamentarium in reconstructive surgeries of the lower limb even in the era of micro and super-microsurgery. It is still a safe and viable option in primary and secondary care centers for the reconstructive surgery of the lower limb in situations such as larger distal defects in the leg and foot where distally based flaps from the ipsilateral leg calf are not feasible.

Even in tertiary care centers cross leg flaps is a viable procedure in failed free flap surgeries. This is quite relevant because the highest percentage of failure of free flaps is encountered in cases of resurfacing the traumatic defects of the distal leg and foot [3].

Like most of the proven procedures, cross leg flaps have also undergone refinement and modifications. With the advent of fasciocutaneous flaps described by Ponten in 1983, the cross leg flaps have been raised safely and easily with $1: 3$ to $1: 3.5$ widths to length ratio [4].

Whenever no possible other options to reconstruct the foot and leg trauma with tissue loss, due to technical deficiency, no available local tissue, and/or loss of healthy donnar vessels for microsurgical reconstruction, the cross-leg flap is the only choice for reconstruction.

This is the aim of my article to address and evaluate this flap and follow up of its results.

\section{Patients and Methods}

This retrospective study was carried upon one hundred thirty five patients suffering from leg and-or foot soft tissue injury who were admitted to emergency department of Sohag University Hospital, and my private clinic 
(late after injury) in the period from April 2009 to June 2011.

The selection criteria were injuries with exposed tendon, bone, or neurovascular structures.

All the patients received 1st aid resuscitative measures to minimize bleeding restore airway and correct shock except those who were not indicated. Detailed history was taken with special emphasis on the mechanism of trauma; the time elapsed since injury, history of previous surgical procedures.

All the patients were subjected to full general and local clinical examination to assess the site and size of the defect, the presence or absence of exposed bone, tendons or neurovascular structures, the degree of wound contamination, the condition of nearby skin, the condition of the contra lateral leg, and full vascular (if there is arterial injury, or distal vascular insufficiency) and neurological examination (to exclude distal nerve affection).

Fresh clean cases are managed primarily, while late or contaminated cases (infection or gangrenous skin) are dealt with secondarily after debridment and granulation have occurred.

Laboratory investigations necessary for surgical fitness were done as complete blood picture, bleeding and clotting time. X-rays, to exclude fracture, emphysema or other orthopedic interference, and Doppler studies, to insure distal vascular adequacy, were done when indicated.

This study focused on the cases that need coverage of bone, tendons, and neurovascular bundle, while those that managed by local flap or split thickness graft were excluded.

Skeletal stability and restoration of the circulation of the injured limb were achieved before or at the session of reconstruction.

Fixation of the two limbs was achieved by strapping, wrapped to both limbs together, in adolescents and calm children, and by cast in younger or naughty children to exclude vigorous movements that may separate the flap.

$1^{\text {st }}$ dressing was done on the $3^{\text {rd }}$ day post operative and twice weekly after that, unless extensive infection is present, daily dressing was done. Separation was achieved after 3 weeks, and at that time any raw areas were splitthickness grafted.

All the patients received postoperative care including proper broad spectrum antibiotic therapy, potent pethedine analgesics in the post-operative period, elevation of the limbs 45 degree to prevent edema and good monitoring of the flap color, temperature and capillary refill.

Ambulation is encouraged early after separation unless there is orthopedic contraindication until fractures heal.

At follow-up, the patients were evaluated for functional (mobility without limping) as well as for cosmetic outcome, also the admission, and follow up sheets were recorded to avoid the remember bias of the patient's family.

The collected data were analyzed using SPSS version 11 for Windows.

\section{Surgical Technique}

The calf was the principle donor site for cross-leg flap, but may be extended distally if a large flap is needed. The choice of location of the base of the flap depends on both the site, size of the defect, and the comfortable position of the leg. Medial and lateral dependant flaps were used according to the position of the defect.

The defect was debrided, and the margin was freshened. After proper preoperative marking, the flap was raised including the fascia, and multiple skin to fascia stitches were taken to prevent any shearing movement to affect the blood supply. Preservation of sural nerve and short saphenus vein was achieved (not included in the flap), if possible. The donor site of the flap was split thickness skin grafted, and the flap was sutured over the defect (Figure 1).

Three weeks later the flap is separated, and any residual raw areas, if present are grafted with split thickness skin graft.

\section{Results}

One hundred thirty five patients suffering from leg andor foot soft tissue injury who were admitted to Sohag University Hospital, and private clinic in the period from April 2009 to June 2011, were included in this study.

Their ages were 2 - 15 years, with mean age $8.8 \pm 3.59$ years, 86 were males, and 49 were females. The most common cause of injury was motor car accident (74\%). This was followed by crush injury (14\%), falling from height (8\%), motor bike (3\%), firearm injury (1\%) (Table 1).

The most common site of injury was the foot (63\%). This was followed by the leg (37\%), and combined leg and foot in (40\%). All cases were with complex wounds with exposed bone, tendons or neurovascular structures.

102 cases had immediate reconstruction within the first 72 hours (75.5\% of cases). 33 cases had delayed

Table 1. Cause of trauma and its percentage.

\begin{tabular}{ccc}
\hline Cause of trauma & Number of patients & $\%$ \\
\hline Motor car accident & 100 & $74 \%$ \\
Crush injury & 19 & $14 \%$ \\
Falling from height & 10 & $8 \%$ \\
Motor bike accident & 4 & $3 \%$ \\
Firearm injury & 2 & $1 \%$ \\
\hline
\end{tabular}



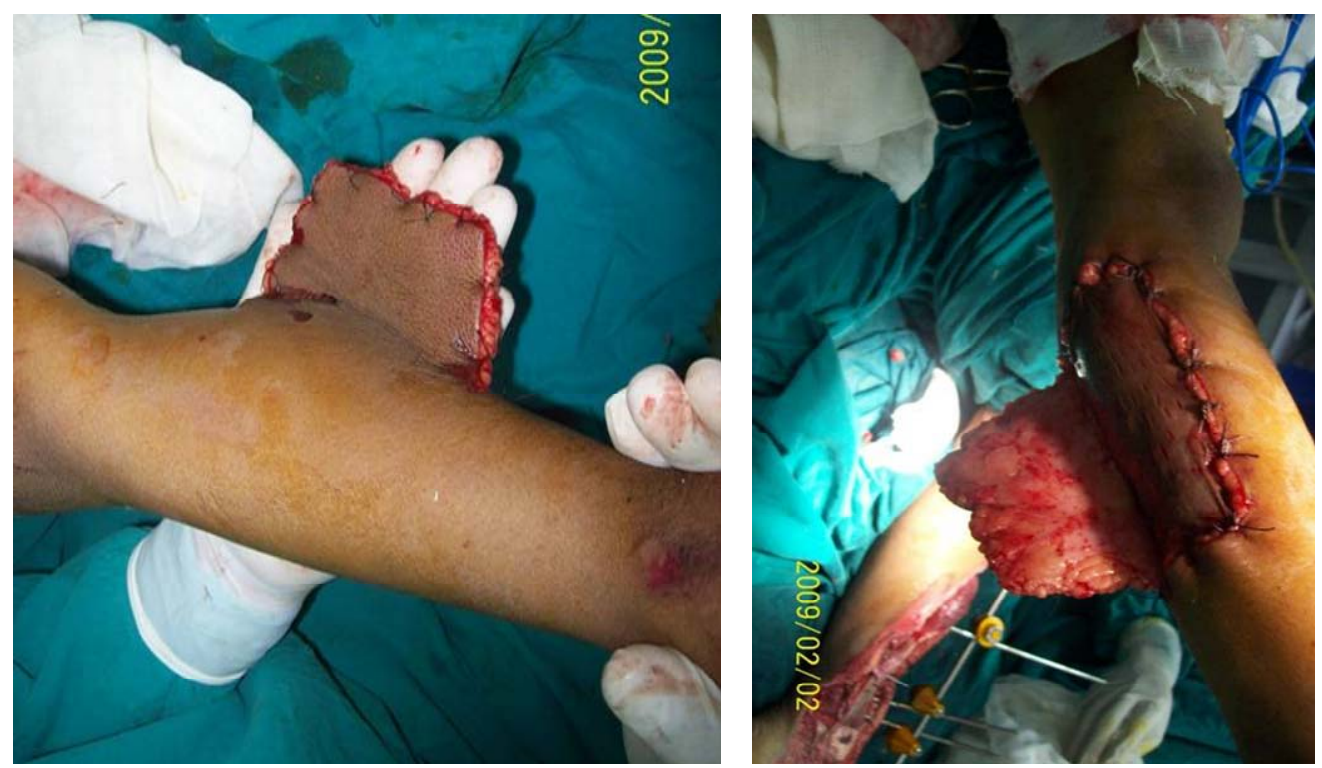

Figure 1. Medially based cross leg flap, right anterior view, and left posterior view with the donnar grafted with split thickness skin graft.

reconstruction (24.5\% of cases).

Follow up for 6 - 18 months with the mean follow up was 12 months \pm 4.74 months.

One hundred twenty three flaps were completely viable after division. Four patients had marginal necrosis, two patients had superficial epidermal necrosis, four patients had dehiscence, and needed repositioning and suturing, but healed well leading to increase the hospital staying, and two patients experienced infection, treated with parentral antibiotic according to culture and sensitivity test, and daily dressing. All healed completely in ten days. Partial graft loss on the flap donor site was seen in seven patients, which required regrafting with split thickness skin graft.

All patients ambulate well after 5 - 10 days, except for 14 patients who had associated orthopedic problems as external fixation in 8 patients, or other fractures in 6 patients.

Cosmetic outcome were excellent in all patients, but 3 patient's mothers complaint of bulky flap, and for them liposuction was done with postoperative satisfaction. 2 cases complaint of early donnar sit graft, but later on they were satisfied (Figures 2-5).

\section{Discussion}

An injury to the lower extremity can be a complex problem, often involving the fractured bone, exposed tendons, and soft tissue defects. Microsurgical free flap is now a well-established procedure in the reconstruction of severely damaged lower extremities. However, successful result depends on the availability of suitable vessel with healthy vascular wall and adequate size for micro vascu- lar anastomosis. Hamilton first introduced the cross-leg flap in 1854. During the Second World War, this flap was used extensively with gratifying results. Stark (1950) standardized the procedure and summarized its usefulness for lower extremities trauma [5].

Free flaps cannot be used in patients with major lower extremity injury with axial vessel damage and a history of previous trauma and thrombosis of vessels. Failed previous free flap presents special problems in reconstruction. Locally diseased arterial tree, recipient vessel not available on exploration, and general condition of the patient not permitting long-standing surgery forms other contraindications for free flap. Relative contraindications of free flap include electrical burns, single vessel limb, delayed referral, and in patients after bone tumor resection that had radiotherapy. In pediatric age group, it is fraught with technical difficulties. In these situations, the cross-leg fasciocutaneous flap can be a good alternative to reconstruct the defects. The indications may be markedly broadened especially in the centers with no access to microsurgery. Hence, the cross-leg flap becomes a valuable option in the aforementioned conditions. Significant donor site deformity and morbidity, long operative hours, and secondary revision for debulking and contouring of the flap specially used around heel and ankle may be considered as additional disadvantages with free tissue transfer [6-9].

In our study, the mean length of time from first operation to complete healing was 35 days, and the mean operating time for both the stages was $1(1 / 2) \mathrm{h}$. Stable coverage was obtained in all the patients. Wells et al. reported that Type IIIB tibial fractures carried a significantly higher risk of free-flap failure than the other types 

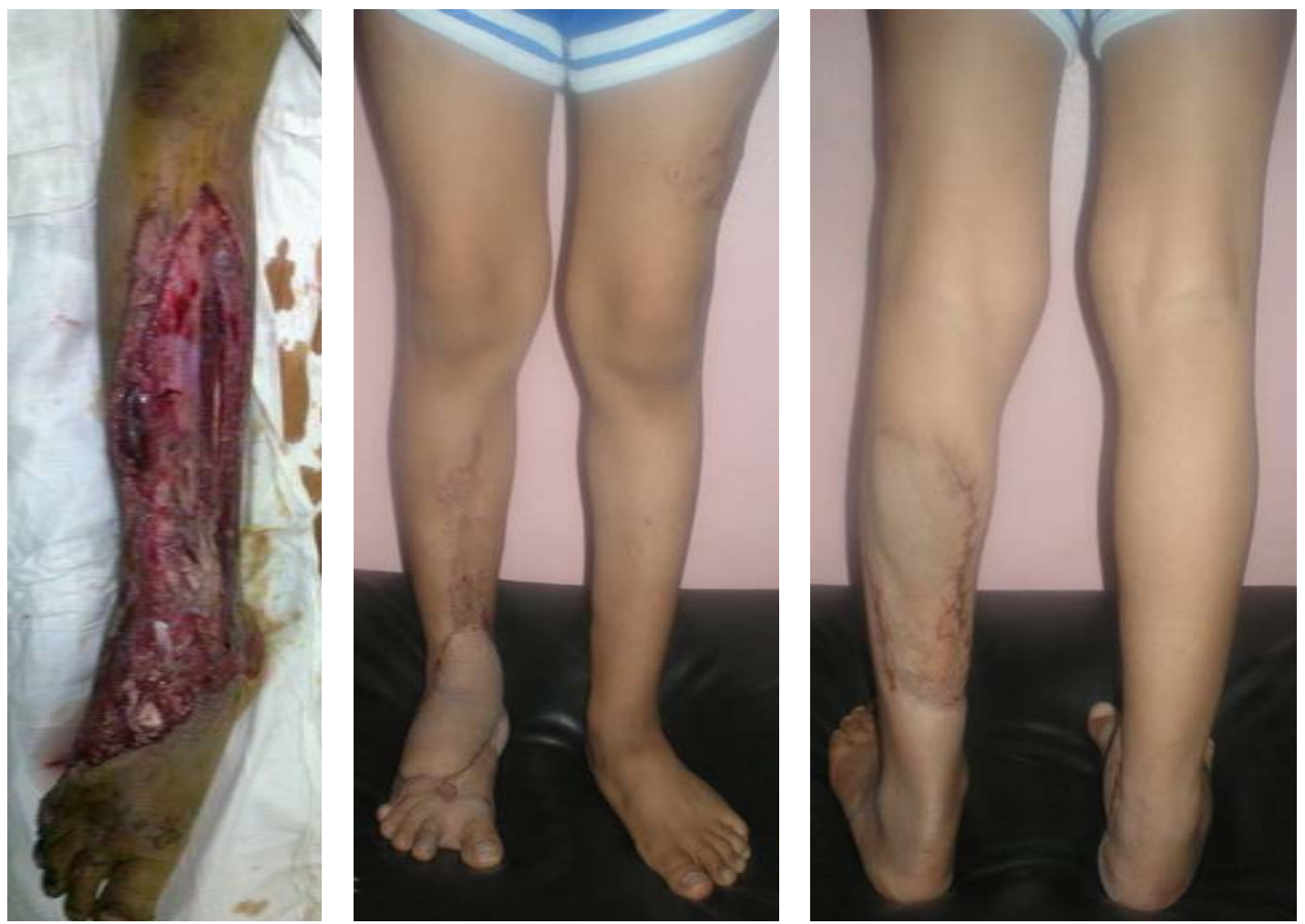

Figure 2. Male patient, 7 years old, with motor car accident left preoperative, middle, and right late post operative, after 8 months.
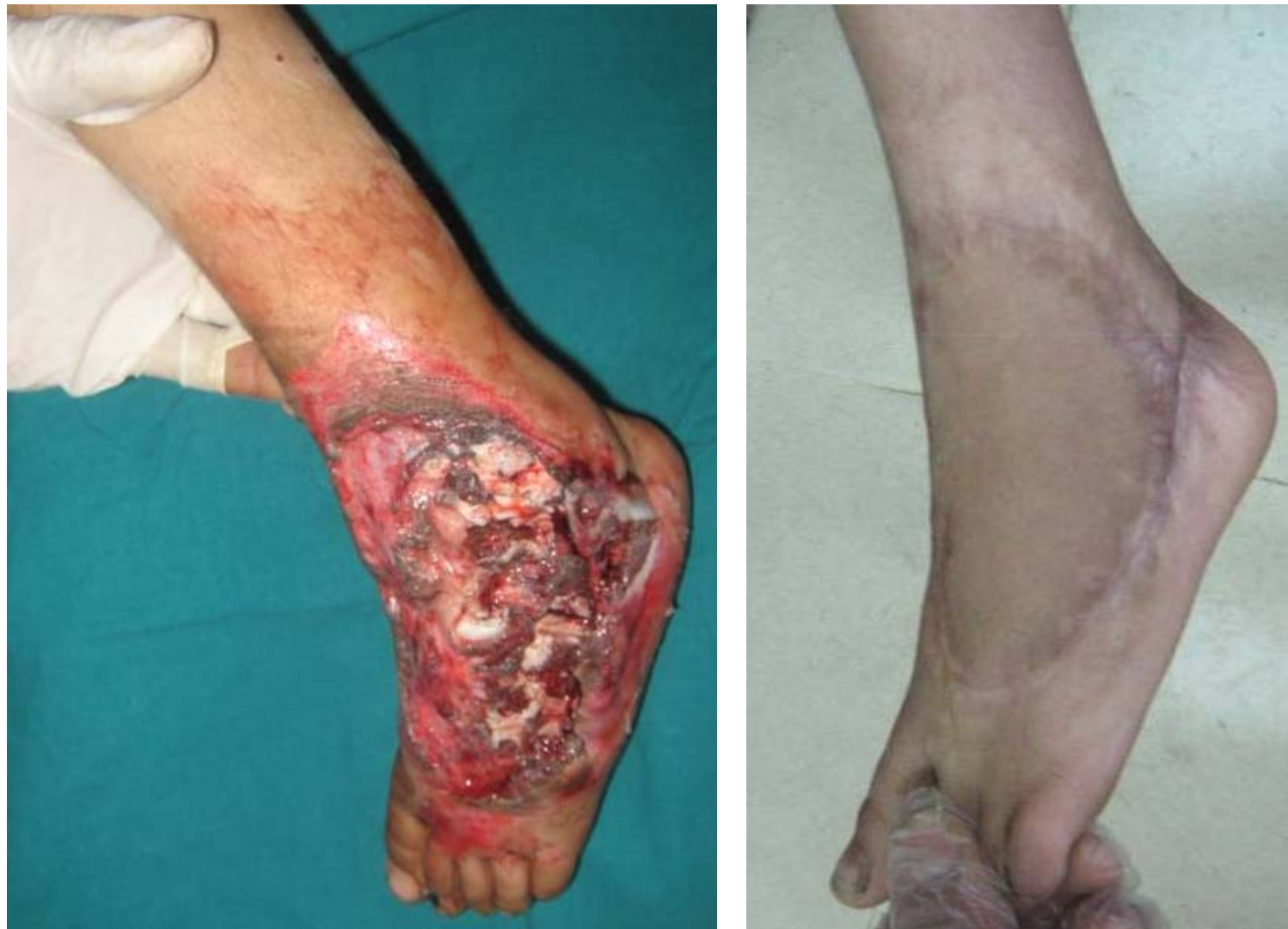

Figure 3. Female patient, 5 years old with crush injury, left preoperative, right one year post operative. 

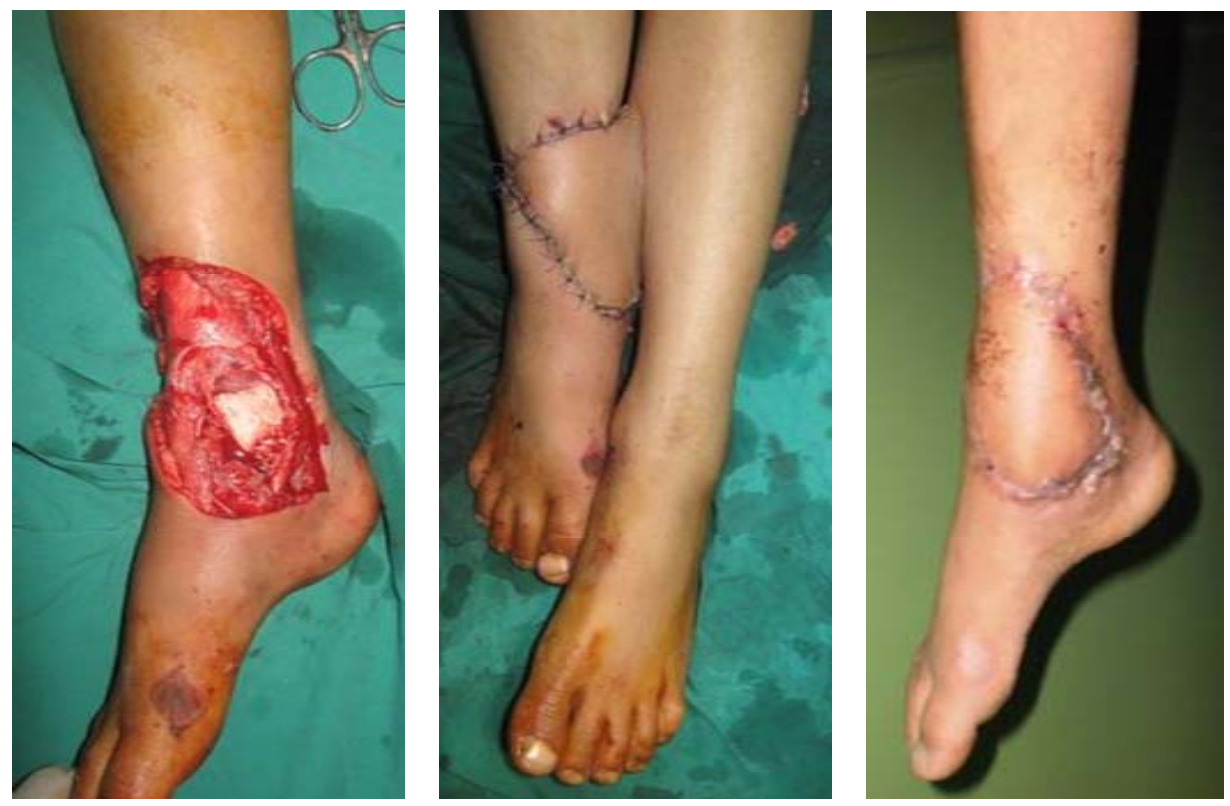

Figure 4. Male patient, 8 years old, left preoperative, middle flap before separation, and right 4 weeks postoperative.
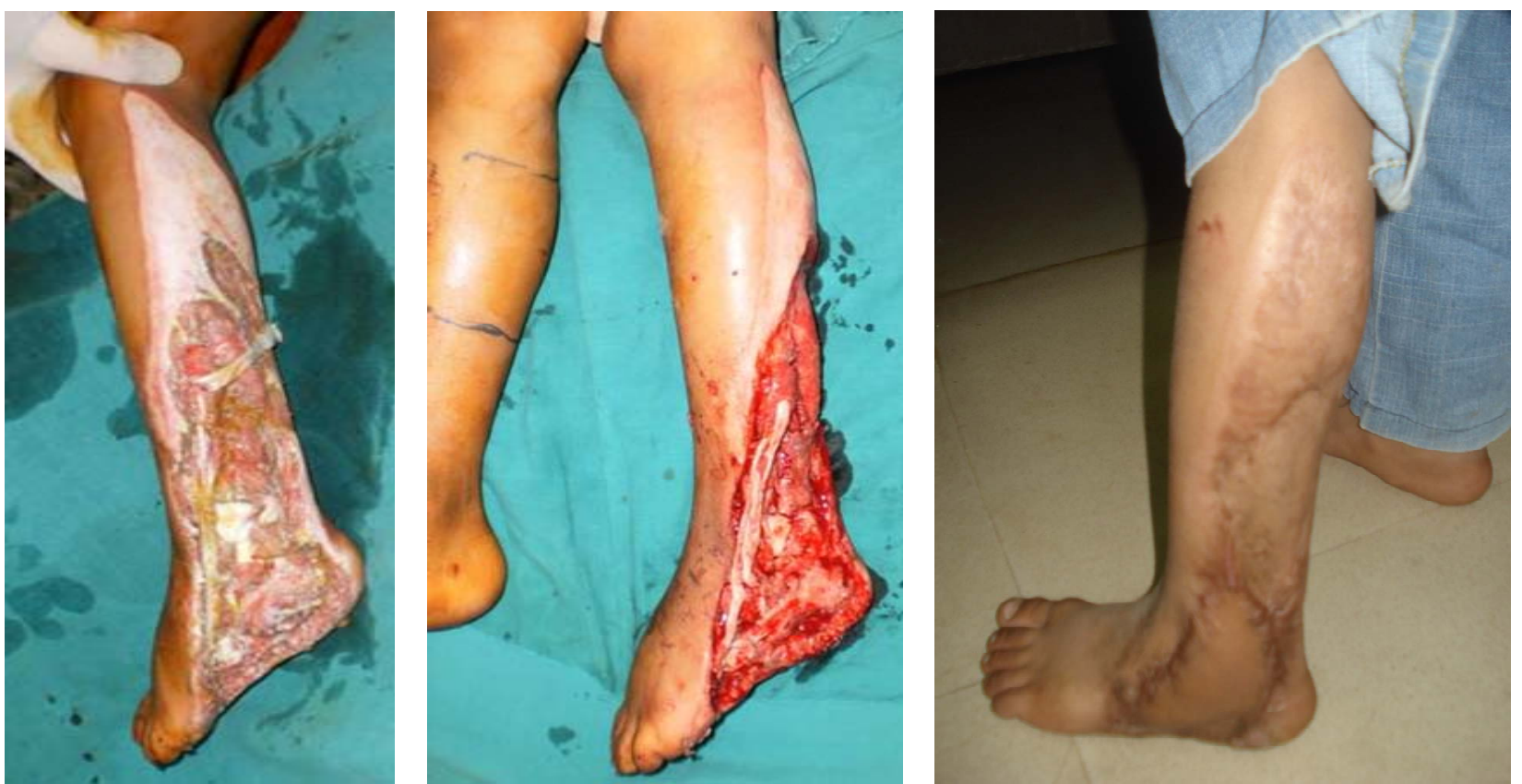

Figure 5. Male patient, 9 years old, left preoperative, middle after debridment, tendon grafting, and right 10 months post operative.

of fracture, and stable, long-term coverage of the free flaps was achieved only in $78 \%$ of patients [10]. For free flap coverage in lower limb, Serafin et al. reported the average time in the hospital as 36.2 days and average operating time as $8 \mathrm{~h}$ [11]. Morris et al. reported 94\% success rate with conventional cross-leg flap, and by incorporating the fascia, the success rate approaches nearly $100 \%$ [12]. For free flap, most of the centers reports success rate in the range of $90 \%-92 \%$ [13].
Cross-leg flaps remain a useful and highly reliable tool for the reconstruction of difficult wounds of the lower limb [14]. It offers the possibility of salvaging limbs that are otherwise nonreconstructable. Cross-extremity flaps function as a nutrient flap for the distal limb even though the pedicle has been divided [15]. It is a backup procedure in an urgent situation and supplies a large quantity of skin. Advantages of cross-leg flap include ease of dissection, versatility, shorter operating time, minimal donor 
site morbidity, and replacement of like tissue with little or no need for secondary revision [16].

With its simplicity, reliability, absence of functional deficit and good-quality coverage with only moderate aesthetic disadvantage, cross-leg flap finds a definite place in reconstructive trauma surgery [17]. Therefore, we recommend its use for injuries unsuitable for local tissue transfer, when real micro vascular expertise is not available or operating room time is restricted.

It is a simple, easy, non time consuming, and had little complication apart from the problem of immobility. In our series, the paucity of complications, and the durability of the flap makes us recommend this reconstructive tool for our patients, and junior plastic surgeons.

\section{Conclusions}

Cross-leg flap offers the possibility of salvaging limbs that are otherwise nonreconstructable.

It is an easy technique, not time consuming, suitable for junior plastic surgeon, and highly reliable tool for the reconstruction of difficult wounds of the lower limbs.

It offers a large flap dimensions to cover most of the defects of the lower extremities especially whenever bone, tendons, and neurovascular bundles are exposed.

\section{REFERENCES}

[1] G. I. Taylor, P. Townsend and R. Corlett, "Superiority of the Deep Circumflex Iliac Vessels as the Supply for Free Groin Flaps: Clinical Work," Plastic and Reconstructive Surgery, Vol. 64, No. 6, 1979, pp. 745-759. doi:10.1097/00006534-197912000-00001

[2] M. Topalan, “A New and Safer Anastomosis Technique in Cross-Leg Flap Procedure Using the Dorsalis Pedis Arterial System," Plastic and Reconstructive Surgery, Vol. 105, No. 2, 2000, pp. 710-713. doi:10.1097/00006534-200002000-00037

[3] B. J. Kamath, T. Varghese and P. Bhard, “A Modified Cross Leg Flap for Large Triangular Defects of the Foot and Ankle," The Foot \& Ankle Journal, Vol. 1, No. 8, 2008, pp. 21-24.

[4] B. Ponten, "The Fasciocutaneous Flap. Its Use in Soft Tissue Defects of Lower Leg," British Journal of Plastic Surgery, Vol. 34, No. 2, 1981, pp. 215-220. doi:10.1016/S0007-1226(81)80097-5

[5] R. B. Stark, “The Cross Leg Flap Procedure,” Plastic and Reconstructive Surgery, Vol. 9, No. 3, 1952, pp. 173-204. doi:10.1097/00006534-195203000-00001

[6] S. Devansh, "Prefabricated Recipient Vascular Pedicle for Free Composite-Tissue Transfer in the Chronic Stage of
Severe Leg Trauma,” Plastic and Reconstructive Surgery, Vol. 96, No. 2, 1995, pp. 392-399. doi:10.1097/00006534-199508000-00020

[7] A. Yamada, K. Harii, K. Ueda, H. Asota and H. Tanaka, "Versatility of a Cross Leg Free Rectus Abdominis Flap for Leg Reconstruction under Difficult and Unfavorable Conditions,” Plastic and Reconstructive Surgery, Vol. 95, No. 7, 1995, pp. 1253-1257. doi:10.1097/00006534-199506000-00017

[8] C. S. Lai, S. D. Lin, C. K. Chou and Y. M. Cheng, "Use of a Cross-Leg Free Muscle Flap to Reconstruct an Extensive Burn Wound Involving a Lower Extremity,” Burns, Vol. 17, No. 6, 1991, pp. 510-513. doi:10.1016/0305-4179(91)90083-S

[9] H. Chen, T. A. El-Gammal, F. Wei, H. Chen, M. S. Noordhoff and Y. Tang, "Cross-Leg Free Flaps for Difficult Cases of Leg Defects: Indications, Pitfalls, and Long-Term Results,” Trauma, Vol. 43, No. 3, 1997, pp. 486-491. doi:10.1097/00005373-199709000-00016

[10] M. D. Wells, C. V. Bowen, R. T. Manktelow, J. Graham and J. B. Boyd, "Lower Extremity Free Flaps: A Review,” Canadian Journal of Surgery, Vol. 39, No. 3, 1996, pp. 233-239.

[11] D. Serafin, N. Georgiade and D. H. Smith, "Comparison of Free Flaps with Pedicled Flaps for Coverage of Defects of the Leg or Foot," Plastic and Reconstructive Surgery, Vol. 59, No. 4, 1977, pp. 492-499.

[12] A. M. Morris and A. C. Buchan, "The Place of the CrossLeg Flap in Reconstructive Surgery of the Lower Leg and Foot: A Review of 165 Cases,” British Journal of Plastic Surgery, Vol. 31, No. 2, 1978, pp. 138-142.

[13] G. G. Hallock, "Impact of the Successful Flap but Failed Reconstruction on the True Rate of Success in Free-Tissue Transfers,” Journal of Reconstructive Microsurgery, Vol. 16, No. 8, 2000, pp. 589-592. doi:10.1055/s-2000-9374

[14] D. J. Hodgkinson and G. B. Irons, "Newer Applications of the Cross-Leg Flap,” Annals of Plastic Surgery, Vol. 4, No. 5, 1980, pp. 381-390. doi:10.1097/00000637-198005000-00005

[15] A. P. Landra, "Salvage of a Seriously Injured Lower Limb with a Musculo-Cutaneous Cross-Leg Flap,” British Journal of Plastic Surgery, Vol. 35, No. 1, 1982, pp. 40 42. doi:10.1016/0007-1226(82)90081-9

[16] D. A. Hudson and K. Millar, "The Cross-Leg Flap: Still a Useful Flap in Children,” British Journal of Plastic Surgery, Vol. 45, No. 2, 1992, pp. 146-149. doi:10.1016/0007-1226(92)90175-W

[17] C. D. Long, M. S. Granick and M. P. Solomon, "The Cross-Leg Flap Revisited,” Annals of Plastic Surgery, Vol. 30, No. 6, 1993, pp. 560-563. doi:10.1097/00000637-199306000-00018 